\title{
Cell Toxicity and inhibitory effects of Cyperus rotundus extract on Streptococcus mutans, Aggregatibacter actinomycetemcomitans and Candida albicans
}

\author{
Moein Khojaste (1), Mohsen Yazdanian (1), Elahe Tahmasebi (1), Mehdi Shokri (2), \\ Behzad Houshmand (3), Razieh Shahbazi (4)
}

(1) Research Center for Prevention of Oral and Dental Diseases, Baqiyatallah University of Medical Sciences, Tehran, Iran; (2). Department of Dental Biomaterials, School of Dentistry, Shahid Beheshti University of Medical Sciences, Tehran, Iran; (3) Department of Periodontics, School of Dentistry, Shahid Beheshti University of Medical Sciences, Tehran, Iran; (4) School of Dentistry, Shahid Beheshti University of Medical Sciences, Tehran, Iran

This article is distributed under the terms of the Creative Commons Attribution Noncommercial License (CC BY-NC 4.0) which permits any noncommercial use, distribution, and reproduction in any medium, provided the original author $(s)$ and source are credited.

\begin{abstract}
Periodontitis and tooth decay are common dental diseases. There are some bacterial risk factors such as Streptococcus mutans, Aggregatibacter actinomycetemcomitans and Candida albicans complications, tubers of Cyperus rotundus was used to determine the antimicrobial effect of fungi. Considering the increasing resistance of bacteria and fungi to antibiotics and their alcoholic and aqueous extracts and essential oil on Streptococcus mutans, Aggregatibacter actinomycetemcomitans and Candida albicans, as well as an examination cytotoxicity on gingival fibroblast cells. Alcoholic and aqueous extracts and essential oil prepared from tubers of Cyperus rotundus. After bacterial and fungal cultures, the inhibition zone, Minimum inhibitory concentrations (MIC), and Minimum Bactericidal Concentration (MBC), were studied. Cytotoxicity was also evaluated using the Methyl tetrazolium test (MTT).The results showed that Cyperus rotundus alcoholic extract had the greatest effect on inhibition of growth and death of Streptococcus mutans, Aggregatibacter actinomycetemcomitans. According to the present study, Cyperus rotundus extract can be considered as a suitable candidate for the treatment and prevention of periodontitis and tooth decay.
\end{abstract}

Key Words: Cyperus rotundus, Periodontitis, Streptococcus mutans, Candida albicans, Aggregatibacter actinomycetemcomitans

Eur J Transl Myol 28 (4): 362-369, 2018

Periodontitis is one of the most common oral diseases and one of the main causes of tooth loss worldwide. ${ }^{1}$ In Periodontitis, periodontal ligament and other tissues that hold the teeth, are invaded by microorganisms. The clinical symptoms includes gingivitis with bleeding, tooth loosening, gum dwindle, unpleasant odor of mouth, alveolar bone destruction and finally tooth loss. Aggregatibacter actinomycetemcomitans is a gramnegative anaerobic bacterium that plays an important role in the development of periodontitis, especially aggressive periodontitis. $^{2}$ Periodontal pockets can easily be saturated with saliva, and are suitable sites for growth of Candida albicans species. ${ }^{3}$ Candida albicans accumulate as biofilm and its proliferation occurs in biofilm at periodontal pockets. Indeed, deep periodontal pockets can alter the balance of subfamily microflora, and then this imbalance leads to colonization of destructive microorganisms in periodontal tissue. ${ }^{4}$ Candida albicans colonizes various parts of the mouth especially caries lesion and dental plaque. Dental caries is a common infectious disease of oral cavity ${ }^{5}$ Several factors are effective in predicting the risk of tooth decay such as oral microbial flora especially Streptococcus mutans, the major microbial cause of tooth decay. Twenty-dive strains of Streptococcus mutans have been discovered, which can destroy the enamel of the tooth by producing lactic acid and sucrose fermentation. ${ }^{5,6}$ One of routine ways to reduce bacterial damages is administration of antibiotics that is accompanied with challenges such as the long course of treatment, side effects and bacterial resistance. Therefore, finding an appropriate replacement 
Effects of Cyperus rotundus extract

Eur J Transl Myol 28 (4): 362-369, 2018

Table 1. Mean of inhibition zone

\begin{tabular}{|r|r|r|r|r|}
\hline & \multicolumn{4}{|c|}{ Mean of inhibition zone } \\
\hline Microorganism & alcoholic extract & aqueous extract & essential oil & chlorhexidine \\
\hline S.mutans & $19.6 \mathrm{~mm}$ & $9.3 \mathrm{~mm}$ & $13.3 \mathrm{~mm}$ & $22.3 \mathrm{~mm}$ \\
\hline $\begin{array}{r}\text { A. actinomycetem } \\
\text { comitans }\end{array}$ & $33.3 \mathrm{~mm}$ & No inhibition zone & $22.3 \mathrm{~mm}$ & $42.3 \mathrm{~mm}$ \\
\hline Calbicans & No inhibition zone & No inhibition zone & No inhibition zone & $25.3 \mathrm{~mm}$ \\
\hline
\end{tabular}

for antibiotic in treatment of periodontitis is particularly importantes. L. Cyperus rotundus from the Cyperaceae family, is a perennial her. ${ }^{7,8}$ Tubers of Cyperus rotundus has terrapin endoperoxide called 10,12-calamenene, a steroid glycoside called sitosterol- $\beta$-D-galactopyranoside and herbal components sucha $\mathrm{s}$ khellin, visnagin, salicylic acid, and p-coumaric acid. ${ }^{9}$ To date, several therapeutic properties have been investigated for Tubers of Cyperus rotundus but the antimicrobial properties and effects of Iranian species of this plant have not been studied yet. Therefore, due to the increased drug resistance of bacteria and side effects of antibiotics and advantages of using herbal medicine, the aim of the present study was to evaluate the inhibitory effect of Essential Oil, alcholic and aqueous extracts of the tubers of Cyperus rotundus and its comparison with Chlorhexidine on Streptococcus mutans, Aggregatibacter actinomycetemcomitans and Candida albicans.

\section{Materials and Methods}

Preparation and Extraction

Tubers of Cyperus rotundus plant was provided from Tehran Agricultural Research Center (Iran) and dried for 2 weeks. 1000 grams of Tubers of Cyperus rotundus were thoroughly crushed. Then $400 \mathrm{ml}$ water was added to 400 $\mathrm{g}$ of crushed plant and the standard hydrodistillation method was performed using Clevenger type apparatus to prepare the essential oil 10. To prepare aqueous and alcholic extracts, separately, $75 \mathrm{~g}$ of the crushed plant was mixed $750 \mathrm{ml}$ of distilled water and pure ethanol and stirred slowly for 72 hours and then separated using filters to obtain initial extracts. The initial extract was introduced into a vacuum distillation apparatus at $80^{\circ} \mathrm{C}$, the solvent evaporated slowly for one hour and the concentrated extract was obtained.

\section{Preparation and culture of microorganisms}

Three microorganisms including $C$. albicans (ATCC 10231), S. mutans (ATCC 35668), and $A$. actinomycetemcomitans (A.a. Jp2 Nov996) were used in this study. Sabouraud dextrose broth (SDA) (Sigma, USA) and BHI agar (Sigma, USA) was used for culture of C. albicans and S. Mutans respectively. Cultures were incubated in aerobic conditions at $37^{\circ} \mathrm{C}$ and after 24 hours, they were cultured on a BHI agar and SDA culture medium to create a single colony and again incubated in aerobic conditions for 24 hours. A. actinomycetem comitans was first cultured for enrichment on the BHI medium (Sigma, USA) under anaerobic conditions (with type A gas pack) for 48 hours at $37{ }^{\circ} \mathrm{C}$ and then was cultured on BHI agar and incubated in anaerobic conditions for 72 hours to create a single colony

\section{Inhibition zone analysis}

In order to evaluate the antibacterial effect of the plant extracts, well diffusion agar method was used. Standard dilutions of microorganism were provided according to MacFarland. ${ }^{11}$ Sterilized swabs were used to prepare suspensions of each microorganism and then to culture them on BHI agar medium. After cultivation, tiny wells were created on BHI agar medium and finally, $50 \mu \mathrm{l}$ of aqueous and alcholic extracts were separately inoculated into the wells. Incubation conditions for $C$.albicans and $S$.mutans were aerobic at $37{ }^{\circ} \mathrm{C}$ for $18-24$ hours, while for $A$. actinomycetemcomitans were anaerobic at $37^{\circ} \mathrm{C}$, for 72 hours. The wells containing distilled water and chlorhexidine were considered as negative and positive controls, respectively. After incubation, the diameter of

Table 2. MIC and MBC test results

\begin{tabular}{|l|l|l|l|}
\hline \multicolumn{4}{|l|}{ S. mutans } \\
\hline Test & alcoholic extract & aqueous extract & essential oil \\
\hline MIC & $1.56 \%$ & $50 \%$ & $6.25 \%$ \\
\hline MBC & $25 \%$ & $100 \%$ & - \\
\hline Test & A. actinomycetemcomitans & \\
\hline MIC & $3.12 \%$ & - & $6.25 \%$ \\
\hline MBC & $12.5 \%$ & - & $12.5 \%$ \\
\hline
\end{tabular}


Effects of Cyperus rotundus extract

Eur J Transl Myol 28 (4): 362-369, 2018

Table 3. Mean cell viability in study groups

\begin{tabular}{|l|l|l|l|}
\hline Group & N & Mean & Std. Deviation \\
& & & \\
& & & \\
\hline Negative Control & 8 & 100.000 & 22.07 \\
\hline Positive Control & 8 & 15.248 & 14.00 \\
\hline chlorohexidine & 8 & $\mathbf{2 0 . 0 2 6}$ & 4.73 \\
\hline Essential oil & 9 & 109.424 & 40.71 \\
\hline Alcoholic extraction & 9 & 150.320 & 51.14 \\
\hline Total & 42 & $\mathbf{8 1 . 4 2 6}$ & $\mathbf{6 1 . 8 5}$ \\
\hline
\end{tabular}

the inhibition zone was measured. The tests were repeated three times to obtain more reliable data.

\section{Determining Minimum Inhibitory Concentrations (MIC) and Minimum Bactericidal Concentration MBC}

Minimum inhibitory concentrations (MIC) test was performed using microdilution and colorimetric methods. Sterilized BHI broth medium $(100 \mu \mathrm{l})$ of was added to each well of the plate. Tubers of Cyperus rotundus extracts $(100 \mu \mathrm{l})$ were added to the first well and then serial dilutions were added to others until the 12th well. Finally, $10 \mu \mathrm{l}$ of diluted McFarland suspension was added into all wells except for negative control, so that the final concentration of the microorganism was 5.55 $\mathrm{cfu} / \mathrm{ml}$. Negative control included extract and BHI broth medium without inoculation of microorganisms and positive control including BHI broth and microorganisms without Cyperus rotundus extracts. This test was repeated three times and performed for each microorganism separately. S. mutans plates were incubated for 18 to 24 hours, and incubation for A. actinomycetemcomitans was in anaerobic conditions for 48 hours. After incubation, 20 $\mu$ of sterilized Resazurin dye (with $0.01 \%$ concentration) (Sigma, USA) was added to each well and then incubated for 2 hours at $37{ }^{\circ} \mathrm{C}$. After incubation, the lowest concentration (blue to pink) was considered as MIC. In order to conduct the Minimum Bactericidal Concentration (MBC) Test, MIC well and a one lower concentration and other higher concentrations were considered. Microorganisms of above wells were cultured on BHI agar, and the lowest concentration, which killed $99 \%$ of the microorganisms, was considered as MBC for $S$.mutans and A. actinomycetemcomitans.

Cytotoxicity testusing Methyl tetrazolium assay (MTT)

Cytotoxicity measurement was performed using Methyl tetrazolium assay (MTT). In order to evaluate the effect of cytotoxicity, MTT test was performed at a higher and a lower concentration than the minimum inhibitory concentration for Tubers of Cyperus rotundus extracts. Samples were divided into 9 groups: 1: negative control containing culture medium. 2: positive control containing distilled water. 3: chlorhexidine (with $0.2 \%$ concentration). 4: Alcoholic extracts at one concentration higher than MIC. 5: Alcoholic extracts at MIC concentration. 6: Alcoholic extracts at one concentration lower than MIC. 7: Essential oil at one concentration higher than MIC. 8: Essential oil at MIC concentration. 9: Essential oil at one concentration lower than MIC. In case of multiple MIC values, the higher value was used in cytotoxicity test.

\section{Cell Culture and treatment}

Human gingival fibroblast cells (HGF) from the Pasteur Institute of Iran were purchased in a $75 \mathrm{~cm} 2$ flask. Cell culture performed on a complete culture medium containing Dulbecco's modified eagle medium (DMEM), (Gibco, USA) supplemented with $10 \%$ heat-inactivated fetal bovine serum (FBS), $100 \mathrm{U} / \mathrm{mL}$ penicillin $\mathrm{G}$ (Sigma, USA) and $100 \mathrm{mg} / \mathrm{mL}$ streptomycin (sigma, USA) and then incubated in $\mathrm{CO} 2$ incubator. After reaching the $80 \%$ confluency, the cells were collected by trypsin and EDTA and centrifuged at $800 \mathrm{~g}$ for $5 \mathrm{~min}$. The supernatant was discarded and $1.5 \times 103$ cells / $\mathrm{ml}$ of HGF cells in a volume of $200 \mu \mathrm{L}$ were seeded in each well of plate. The plate was stored at $37^{\circ} \mathrm{C}$ for 24 hours. The supernatant was then discarded and washed with PBS. $100 \mu \mathrm{l}$ of MTT was added to all wells in dark place. The cells then returned to the $\mathrm{CO} 2$ incubator to view the Formazan crystals by inverted microscope. The supernatant was discarded and $100 \mu \mathrm{l}$ DMSO (Sigma, USA) was added to each well. Then the absorbance of each well was read at 570 and $620 \mathrm{~nm}$ using ELISA reader. The percentage of subtraction of the absorbed cells affected by the drug was divided to absorption of the control cell as the percentage of cells that remained stable.

\section{Statistical analysis}

Data was first analyzed by SPSS v.20 program. Analysis of variance and Tukey's range test were used to 
Table 4-A. Comparison of cytotoxicity between study groups

\begin{tabular}{|c|c|c|c|}
\hline Group & Group & Mean Difference & Significance \\
\hline \multirow[t]{8}{*}{ Negative Control } & Positive Control & 84.75131* $^{*}$ & .001 \\
\hline & CLX & 79.97382* & .001 \\
\hline & EO High & -3.83944 & 1.000 \\
\hline & EO Medium & -12.91449 & 1.000 \\
\hline & EO Low & -11.51832 & 1.000 \\
\hline & Alcohol High & -63.52531 & .167 \\
\hline & Alcohol Medium & -44.67714 & .593 \\
\hline & Alcohol Low & -42.75742 & .646 \\
\hline \multirow[t]{8}{*}{ Positive Control } & Negative Control & $-84.75131^{*}$ & .001 \\
\hline & CLX & -4.77749 & 1.000 \\
\hline & EO High & $-88.59075^{*}$ & .013 \\
\hline & EO Medium & $-97.66579 *$ & .005 \\
\hline & EO Low & $-96.26963^{*}$ & .006 \\
\hline & Alcohol High & $-148.27661^{*}$ & .000 \\
\hline & Alcohol Medium & $-129.42845^{*}$ & .000 \\
\hline & Alcohol Low & $-127.50873^{*}$ & .000 \\
\hline \multirow[t]{8}{*}{ CLX } & Negative Control & $-79.97382^{*}$ & .001 \\
\hline & Positive Control & 4.77749 & 1.000 \\
\hline & EO High & $-\mathbf{8 3 . 8 1 3 2 6}{ }^{*}$ & .023 \\
\hline & EO Medium & $-92.88831^{*}$ & .008 \\
\hline & EO Low & $-91.49215^{*}$ & .010 \\
\hline & Alcohol High & $-143.49913^{*}$ & .000 \\
\hline & Alcohol Medium & $-124.65096^{*}$ & .000 \\
\hline & Alcohol Low & $-122.73124^{*}$ & .000 \\
\hline & 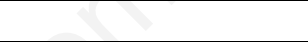 & & \\
\hline \multirow[t]{8}{*}{ EO High } & Negative Control & 3.83944 & 1.000 \\
\hline & Positive Control & $88.59075^{*}$ & .013 \\
\hline & CLX & $83.81326 *$ & .023 \\
\hline & EO Medium & -9.07504 & 1.000 \\
\hline & EO Low & -7.67888 & 1.000 \\
\hline & Alcohol High & -59.68586 & .460 \\
\hline & Alcohol Medium & -40.83770 & .861 \\
\hline & Alcohol Low & -38.91798 & .889 \\
\hline
\end{tabular}

demonstrate differences between observed variables. $\mathrm{P}$ value of $<0.05$ used to indicate statistical significance.

\section{Results}

Antibacterial properties of Tubers of Cyperus rotundus extracts

Inhibition zone for $S$.mutans and $A$. actinomycetemcomitans was the highest in the $0.2 \%$ chlorhexidine group $22 \mathrm{~mm}$ and $42 \mathrm{~mm}$ diameters respectively. In the case of $S$. mutans, inhibition zone in presence of the alcoholic extract, essential oil and aqueous extract was $19 \mathrm{~mm}, 14 \mathrm{~mm}$ and $10 \mathrm{~mm}$ respectively. Aqueous extract had no inhibitory effect on growth of $A$. actinomycetemcomitans, while a diameter of $42 \mathrm{~mm}$ for chlorhexidine, $35 \mathrm{~mm}$ for alcoholic extract and $23 \mathrm{~mm}$ for essential oil observed. Also, none of alcoholic extract, aqueous extracts and essential oil, had no effect on C. albicans. Therefore, MIC and MFC tests for $C$. albicans were not performed. Results of inhibition zone have been demonstrated in table 1. For microbial tests, the highest concentrations available in the aqueous extract and alcoholic extract of $500 \mathrm{mg} / \mathrm{ml}$ were used and the subsequent dilutions were prepared using this concentration. In the study of minimum inhibitory and 
Table 4-B. Comparison of cytotoxicity between study groups

\begin{tabular}{|c|c|c|c|}
\hline \multirow[t]{8}{*}{ EO Medium } & Negative Control & 12.91449 & 1.000 \\
\hline & Positive Control & 97.66579* & .005 \\
\hline & CLX & 92.88831* & .008 \\
\hline & EO High & 9.07504 & 1.000 \\
\hline & EO Low & 1.39616 & 1.000 \\
\hline & Alcohol High & -50.61082 & .668 \\
\hline & Alcohol Medium & -31.76265 & .963 \\
\hline & Alcohol Low & -29.84293 & .974 \\
\hline \multirow[t]{8}{*}{ EO Low } & Negative Control & 11.51832 & 1.000 \\
\hline & Positive Control & $96.26963^{*}$ & .006 \\
\hline & CLX & $91.49215^{*}$ & .010 \\
\hline & EO High & 7.67888 & 1.000 \\
\hline & EO Medium & -1.39616 & 1.000 \\
\hline & Alcohol High & -52.00698 & .636 \\
\hline & Alcohol Medium & -33.15881 & .952 \\
\hline & Alcohol Low & -31.23909 & .966 \\
\hline \multirow[t]{8}{*}{ Alcohol High } & Negative Control & 63.52531 & .167 \\
\hline & Positive Control & 148.27661* & .000 \\
\hline & CLX & $143.49913^{*}$ & .000 \\
\hline & EO High & 59.68586 & .460 \\
\hline & EO Medium & 50.61082 & .668 \\
\hline & EO Low & 52.00698 & .636 \\
\hline & Alcohol Medium & 18.84817 & .999 \\
\hline & Alcohol Low & 20.76789 & .998 \\
\hline \multirow[t]{8}{*}{ Alcohol Medium } & Negative Control & 44.67714 & .593 \\
\hline & Positive Control & $129.42845^{*}$ & .000 \\
\hline & CLX & $124.65096 *$ & .000 \\
\hline & EO High & 40.83770 & .861 \\
\hline & EO Medium & 31.76265 & .963 \\
\hline & EO Low & 33.15881 & .952 \\
\hline & Alcohol High & -18.84817 & .999 \\
\hline & Alcohol Low & 1.91972 & 1.000 \\
\hline \multirow[t]{8}{*}{ Alcohol Low } & Negative Control & 42.75742 & .646 \\
\hline & Positive Control & $127.50873^{*}$ & .000 \\
\hline & CLX & $122.73124^{*}$ & .000 \\
\hline & EO High & 38.91798 & .889 \\
\hline & EO Medium & 29.84293 & .974 \\
\hline & EO Low & 31.23909 & .966 \\
\hline & Alcohol High & -20.76789 & .998 \\
\hline & Alcohol Medium & -1.91972 & 1.000 \\
\hline
\end{tabular}

bactericidal concentration, the lowest concentration with the ability to inhibit growth in $S$. mutans was related to $1.56 \%$ alcoholic extract, which is $6.25 \%$ in essential oil and $50 \%$ in aqueous extracts. The minimum concentration of MBC was also observed in alcoholic extract at $25 \%$ concentration followed by aqueous extract at a concentration of $100 \%$ (Table 2). In $A$. actinomycetemcomitans, the lowest concentration of MIC was $3.12 \%$ for alcoholic extract and $6.25 \%$ for essential oil. The MBC content of alcoholic extract and essential oil was obtained at $12.5 \%$ concentration (Table 2).

\section{Cell cytotoxicity}

After observing the more suitable antimicrobial effect of the alcoholic extract and essential oil, MTT assay were evaluated for HGF cells to investigate the possible cytotoxic properties. For this purpose, alcoholic extracts and essential oil were obtained at higher concentrations and lower than the MIC, and their cytotoxic effects were compared with $0.2 \%$ chlorhexidine over a 24 hour period. 


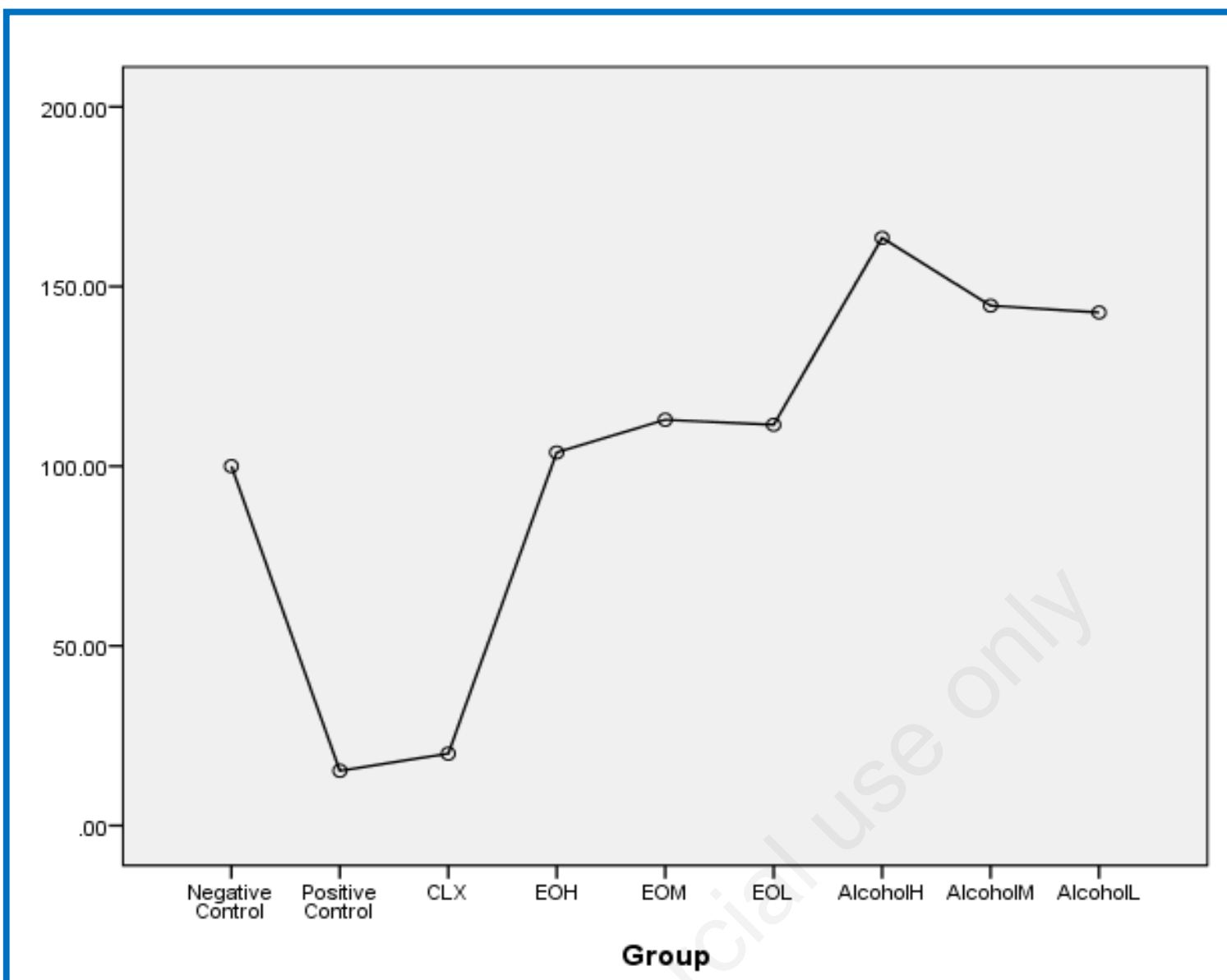

Fig 1. The viability of cells in each of the groups examined and compared with each other.

Based on the toxic effects on the cells, there was a significant difference between the groups of alcoholic extract, essential oil and chlorohexidine and positive control $(p$ value $=0.000)$. chlorohexidine had the highest toxic effect on the cells with mean $20.02 \pm 4.7$. Results of MTT showed that there was a significant difference in these groups with positive control $(\mathrm{P}<0.05)$. (Table 4, A and $\mathrm{B})$. However, there was no significant difference between chlorohexidine and positive control ( $\mathrm{p}$ value $=$ 1.000). Also, there was a significant difference between the treated cells using essential oil and alcoholic extracts with chlorohexidine $(p<0.05)$ for both groups at all concentrations. However, there was no significant difference between the essential oil and alcoholic extract ( $\mathrm{p}$ value $>0.05$ ) and both treatments showed low toxicity on the cells after 24 hours (Table 4, A and B). Figure 1 shows the viability of the cells in each of the groups examined and compared with each other.

\section{Discussion}

A. actinomycetemcomitans is one of the most important causes of periodontal disease. ${ }^{12,13}$ The main treatment of this disease is scaling and root palnning and ultrasonic debridement to remove bacterial deposits. S. mutans is the most important bacteria causing dental caries.
Therefore, the use of mechanisms to reduce these microorganisms is essential. ${ }^{14}$ The aim of the present study was to investigate the effects of ethalonic and aqueous extracts and essential oil of Tubers of Cyperus rotundus on $S$. mutans, A. actinomycetemcomitans and $C$. albicans. The results of inhibition zone in this study, using statistical analyzes, showed a significant difference in growth of $S$. mutans, A. actinomycetemcomitans under the influence of ethalonic and aqueous extracts and essential oil of Tubers of Cyperus rotundus. In this study, alcholic extract of Tubers of Cyperus rotundus in very low concentrations $(7.18 \mathrm{mg} / \mathrm{ml}$ and $15.62 \mathrm{mg} / \mathrm{ml}$ ) has inhibitory effects on $\mathrm{S}$. mutans and $A$. actinomycetemcomitans. In terms of toxicity effect, alcholic extracts of Tubers of Cyperus rotundus in 31.25 $\mathrm{mg} / \mathrm{ml}$ concentration and essential oil in $6.25 \%$ volume concentration showed the lowest cytotoxicity. Chlorohexidine $0.2 \%$ showed the highest cytotoxicity after 24 hours compared to the control group ( $\mathrm{p}$ value $=$ 0.01). This comparison suggests that different concentrations of alcholic extract and essential oil have no cytotoxic, while chlorhexidine has a $100 \%$ toxicity for cells. Antibacterial effects of extracts of Tubers of Cyperus rotundus have been observed in previous studies. However this effect on $A$. 
actinomycetemcomitans as one of the most important factors involved in periodontal disease, has not been studied yet. Somayeh Kilani et al., Studied the antibacterial effects of essential oil of Tubers of Cyperus rotundus, on Staphylococcus aureus, Enterococcus faecalis, Escherichia coli, Salmonella enteritidis and Salmonella typhimurium, and concluded that the oil extracted from this plant has a strong inhibitory effect on S. aureus and E. faecalis and a moderate inhibitory effect on $S$. typhimurium. ${ }^{15}$ In another study, Yu et al., investigated the effects of Tubers of Cyperus rotundus extracts on $S$. mutans. The results showed that the extract was effective in inhibiting the growth of this bacteria and its acid production ${ }^{16}$ Sharma and colleagues evaluated the effects of aqueous, alcholic, chloroform and ether extracts of Tubers of Cyperus rotundus on E. coli, Staphylococcus epidermidis, Bacillus cereus, Pseudomonas aeruginosa, Aspergillus niger and $C$. albicans. They concluded that alcholic extract had the highest inhibitory effect on B.cereus, S. epidermium and $P$. aeruginosa respectively. None of the extracts had inhibitory effect on E.coli and C. albicans. ${ }^{17}$ These results are in concordance with our study, as we found that none of the aqueous, alcholic extracts and essential oil, had no inhibitory effect on $C$. albicans and the best antibacterial effect, and was due to alcoholic extract. Also, unlike other studies, in the present study, we used essential oil of Tubers of Cyperus rotundus to evaluate and compare its inhibitory and antibacterial effects with aqueous and alcoholic extracts. Our results demonstrated its antibacterial effect, although in comparison with alcoholic extract, this compound has less inhibitory effect. In the present study, the effects of aqueous extract of Tubers of Cyperus rotundus on $S$. mutans was observed only at a high concentration of $500 \mathrm{mg} / \mathrm{ml}$, but did not have any inhibitory effects on $A$. actinomycetemcomitans. Results of cytotoxicity test suggested that alcoholic extract and essential oil not only have no toxic effects on HGF cells, but also could have proliferative effects. According to this study, it seems that the alcoholic extract of Tubers of Cyperus rotundus can be a potential candidate for mouthwash in the future. However, more laboratory and clinical studies are needed to confirm the results of this study. In conclusion, the results of this study show that extracts of Tubers of Cyperus rotundus have a good antibacterial effect and can be used as a treatment option in dental infections.

\section{List of acronyms}

HGF - Human gingival fibroblast cells MBC - Minimum Bactericidal Concentration

MIC -Minimum inhibitory concentrations

MTT - Methyl tetrazolium test

SDA - Sabouraud dextrose broth

\section{Author's contributions}

MY, ET, MSH, BH, RSH, and MKH equally participated in experimental design, data collection, writing and revision of the manuscript.

\section{Acknowledgments None.}

Funding This research was supported by Emam Khomeyni Dental Clinic and Shahid Shokri Dental Hospital of Baqiyatallah University of Medical Sciences.

\section{Conflict of Interest}

The authors report no conflicts of interests.

\section{Ethical Publication Statement}

We confirm that we have read the Journal's position on issues involved in ethical publication and affirm that this report is consistent with those guidelines.

\section{Corresponding Author}

Mohsen Yazdanian, Research Center for Prevention of Oral and Dental Diseases, Baqiyatallah University of Medical Sciences, Tehran, Iran

Tel: 00989122163963

E-mail: drmyazdanian@yahoo.com

E-mails of co-authors

Moein Khojaste: Moein.khojaste@yahoo.com

Elahe Tahmasebi: Elahe.tahmasebi.delfan@gmail.com

Mehdi Shokri:Mehdyshokri@gmail.com

Behzad Houshmand:Houshmandperio@ rocketmail.com RaziehShahbazi: Shahbazyrazieh@yahoo.com

\section{References}

1. Parisotto TM, Steiner-Oliveira C, Duque C, et al. Relationship among microbiological composition and presence of dental plaque, sugar exposure, social factors and different stages of early childhood caries. Archives of Oral Biology 2010 ;55:365-73.

2. Henderson B, Ward JM, Ready D. Aggregatibacter (Actinobacillus) actinomycetemcomitans: a triple A* periodontopathogen?. Periodontology 2000. 2010;54:78-105.

3. Sztukowska MN, Dutton LC, Delaney C, et al. Community Development between Porphyromonas gingivalis and Candida albicans Mediated by InlJ and Als3. MBio 2018;9:45-49.

4. Vieira Colombo AP, Magalhaes CB, Hartenbach FA, et al. Periodontal-disease-associated biofilm: A reservoir for pathogens of medical importance. Microbial Pathogenesis 2016;94:27-34.

5. Forssten SD, Bjorklund $\mathrm{M}$, Ouwehand AC. Streptococcus mutans, caries and simulation models. Nutrients 2010;2:290-8.

6. Hamada S, Koga T, Ooshima T. Virulence factors of Streptococcus mutans and dental caries prevention. Journal of Dental Research. 1984;63:407-11. 


\section{Effects of Cyperus rotundus extract}

Eur J Transl Myol 28 (4): 362-369, 2018

7. Prabhakar J, Balagopal S, Priya MS, et al. Evaluation of antimicrobial efficacy of Triphala (an Indian Ayurvedic herbal formulation) and $0.2 \%$ chlorhexidine against Streptococcus mutans biofilm formed on tooth substrate: An in vitro study. Indian Journal of Dental Research 2014;25:475.

8. Kilani S, Abdelwahed A, Ammar RB, et al. Chemical composition, antibacterial and antimutagenic activities of essential oil from (Tunisian) Cyperus rotundus. Journal of Essential Oil Research 2005;17:695-700.

9. Fabricant DS, Farnsworth NR. The value of plants used in traditional medicine for drug discovery. Environ Health Perspective 2001: 109: 69-75.

10. Bousbia N, Vian MA, Ferhat MA, et al. Comparison of two isolation methods for essential oil from rosemary leaves: Hydrodistillation and microwave hydrodiffusion and gravity. Food Chemistry 2009;114:355-62.

11. Balouiri M, Sadiki M, Ibnsouda SK. Methods for in vitro evaluating antimicrobial activity: A review. Journal of Pharmaceutical Analysis 2016;6:71-9.

12. Schacher B, Baron F, Roßberg $M$, et al. Aggregatibacter actinomycetemcomitans as indicator for aggressive periodontitis by two analysing strategies. Journal of Clinical Periodontology 2007;34:566-73.
13. Casarin R, Ribeiro ÉDP, Mariano F, et al. Levels of Aggregatibacter actinomycetemcomitans, Porphyromonas gingivalis, inflammatory cytokines and species-specific immunoglobulin $\mathrm{G}$ in generalized aggressive and chronic periodontitis. Journal of Periodontal Research 2010;45:635-42.

14. Loesche WJ, Grossman NS. Periodontal disease as a specific, albeit chronic, infection: diagnosis and treatment. Clinical Microbiology Reviews 2001 ;14:727-52.

15. Kilani S, Abdelwahed A, Ammar RB, et al. Chemical composition, antibacterial and antimutagenic activities of essential oil from (Tunisian) Cyperus rotundus. Journal of Essential Oil Research $2005 ; 17: 695-700$.

16. Yu HH, Lee DH, Seo SJ, You YO. Anticariogenic properties of the extract of Cyperus rotundus. The American journal of Chinese medicine 2007;35:497-505.

17. Sharma SK, Singh AP. Antimicrobial investigations on rhizomes of Cyperus rotundus Linn. Der Pharmacia Letter 2011;3:427-31.

Received for publication: 06/11/2018

Accepted for publication: 12/11/2018 